\title{
The Electrostatic Interaction Force between a Charge-Regulated Particle and a Rigid Surface
}

\author{
Jyh-PING $\mathrm{Hsu}^{1}$ AND Yung-ChiH KuO \\ Department of Chemical Engineering, National Taiwan University, Taipei, Taiwan 10617, Republic of China
}

Received January 17, 1996; accepted May 7, 1996

The electrostatic interaction force between a charge-regulated particle covered by an ion-penetrable charged membrane and a rigid charged surface immersed in a mixed $(a: b)+(c: b)$ electroIyte solution is analyzed. The membrane contains both acidic and basic functional groups, and a general form for each is assumed which allows multiple proton transfer among functional groups. The rigid surface is negatively charged, and the net charges carried by the particle is also negative. We show that if the separation distance between the particle and the surface is greater than a critical value, the multivalent cations present in the liquid phase have the effect of reducing the repulsion force between the particle and the surface. The reverse is true if the separation distance is smaller than the critical value. For a constant total number of functional groups in the membrane phase, if the membrane is sufficiently thin, the variation of repulsion force as a function of separation distance exhibits a maximum. 1996 Academic Press, Inc.

Key Words: electrolyte solution; mixed; interaction force, electrostatic, repulsive; particle, charge-regulated; ion-penetrable membrane; functional groups, acid and basic, multiple proton transfer; Poisson- Boltzmann equation, nonlinear, numerical solution.

\section{INTRODUCTION}

The deposition of cells to surfaces is a phenomenon common to various biological processes. One of the key measures to this phenomenon is the rate of deposition, which is a function of the interaction forces between cell and surface. As pointed out by Weiss and Harlos (1), interaction forces between cell and surface other than purely gravitational force exist during the course of cell deposition. One of the possible forces is that due to the electrostatic interaction, which depends largely on the charged conditions at both surfaces. Compared with lifeless entities, the electrical condition at cell surface is complicated; it is determined by the degree of dissociation of the functional groups it bears. This leads to the so-called

${ }^{1}$ To whom correspondence should be addressed. charge-regulation phenomenon. Ninham and Parsegian (2) initiated the analysis by proposing a model in which the surface of an entity contains single ionizable functional groups to simulate the charge-regulation behavior of a biological cell. The degree of dissociation of these functional groups varies with the electrical condition of the surrounding medium. The charge-regulation behavior was interpreted as that two interacting surfaces tend to minimize the total interaction free energy (3). The model of Ninham and Parsegian was modified by Chan et al. (4, 5 ) to the case of amphoteric functional groups, by Prieve and Ruckenstein (6) to multiple ionizable functional groups, and by Ohshima and Ohki (7) to a particle composed of a charge-free core and an ion-penetrable membrane bearing fixed charges, which arise from the dissociation of the functional groups distributed in the membrane. In the last case the functional groups are spread over a finite volume in space, rather than over a rigid surface. An example in practice is human erythrocytes, the peripheral zone of which contains a glycoprotein layer about 15 $\mathrm{nm}$ thick which possesses some ionogenic groups, and forms the outer boundary of the lipid layer $(8,9)$. Previous efforts for the core-membrane model are mainly limited to those for the case of low electrical potential and single symmetric electrolyte to allow for a simpler mathematical treatment. Furthermore, the number of protons that an acidic functional group can release, or that a basic functional group can absorb is almost always assumed as unity. These are inconsistent, in general, with those observed in practice.

In the present study, the electrostatic interaction force between a particle, which simulates a biological cell, and a charged surface is analyzed. The core-membrane model of Ohshima and Ohki (7) is extended to the case in which both acidic and basic functional groups are present in the membrane phase, and a general form for each is assumed which allows for multiple proton transfer among functional groups. A mixed $(a: b)+(c: b)$ electrolyte solution for examining the effect of the multivalent cations on the electrostatic interaction force is considered. 


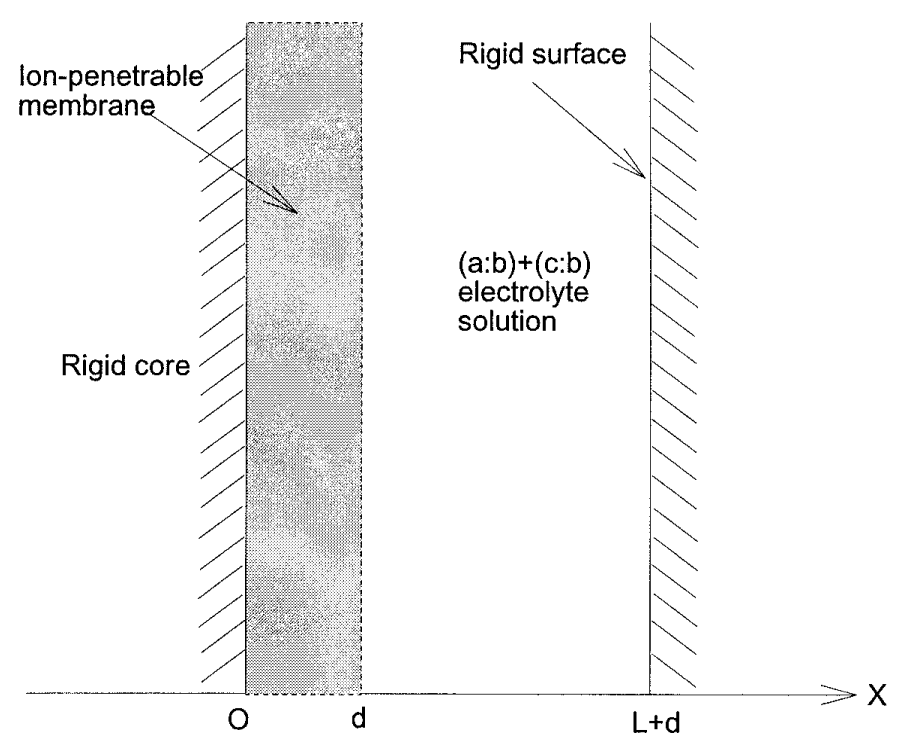

FIG. 1. Schematic representation of the system under consideration. The membrane has a dimensionless thickness $d$. The dimensionless distance between the membrane and the rigid surface is $L$.

\section{ANALYSIS}

By referring to Fig. 1, we consider a planar particle comprised of a rigid core and an ion-penetrable membrane and a rigid, charged surface immersed in a mixed $(a: b)+(c: b)$ electrolyte solution. The particle and the surface are parallel to each other with the former located at the origin, and the latter at $(L+d), d$ and $L$ being the dimensionless thickness of the membrane and the dimensionless distance between the membrane and the rigid surface, respectively. The membrane bears fixed charges due to the dissociation of the functional groups it contains; both acidic and basic functional groups are present. Suppose that these functional groups are distributed uniformly.

\section{Electrical Potential Distribution}

For the present case, the electrical potential distribution is governed by the Poisson-Boltzmann equation

$$
\begin{aligned}
\frac{d^{2} \psi}{d X^{2}} & =\frac{1}{(a+b)+(c-a) \eta} \\
& \times\left\{\left[e^{b \psi}-(1-\eta) e^{-a \psi}-\eta e^{-c \psi}\right]+i N\right\}, i=1,2
\end{aligned}
$$

where $\psi=e \phi / k_{\mathrm{B}} T, X=\kappa r, \eta=c n_{c}^{0} / b n_{b}^{0}, b n_{b}^{0}=\left(a n_{a}^{0}+\right.$ $\left.c n_{c}^{0}\right), \kappa^{2}=e^{2}\left[a(a+b) n_{a}^{0}+c(b+c) n_{c}^{0}\right] / \varepsilon_{0} \varepsilon_{\mathrm{r}} k_{\mathrm{B}} T$, and $N$ $=N_{\mathrm{A}}\left(N_{-}^{i=1}-N_{+}^{i=1}\right) / b n_{b}^{0}$. Here, $r$ denotes the position variable; $\phi$ is the electrical potential; $\eta$ is the fraction of cation with valence $c$ in the bulk liquid phase; $n_{a}^{0}, n_{b}^{0}$, and $n_{c}^{0}$ are, respectively, the number concentrations of ion species with valences $a,-b$, and $c$ in the bulk liquid phase; $\kappa$ is the reciprocal Debye length; $e$ and $N_{\mathrm{A}}$ are, respectively, the elementary charge and the Avogadro number; $\varepsilon_{\mathrm{r}}$ and $\varepsilon_{0}$ are the relative permittivity of liquid phase and the permittivity of a vacuum, respectively; $k_{\mathrm{B}}$ is the Boltzmann constant; $T$ is the absolute temperature; $N_{-}^{i=1}$ and $N_{+}^{i=1}$ are, respectively, the densities of the negative and the positive fixed charges in the membrane phase; and $i$ is a region index $(i=0$ represents the double-layer region, $i=1$ denotes the membrane phase). We assume that the boundary conditions associated with [1] are

$$
\begin{gathered}
\left(\frac{d \psi}{d X}\right)_{x \rightarrow 0} \rightarrow 0, \\
\left(\frac{d \psi}{d X}\right)_{X \rightarrow d^{-}}=\left(\frac{d \psi}{d X}\right)_{X \rightarrow d^{+}}, \\
\psi_{X \rightarrow d^{-}}=\psi_{X \rightarrow d^{+}}, \\
\psi=\psi_{0} \text { at } \quad X=L+d,
\end{gathered}
$$

where $\psi_{0}$ is the dimensionless electrical potential at the rigid surface.

\section{Dissociation of Functional Groups}

We assume that two types of functional groups are present in the membrane phase, and each assumes a general form. The dissociation of these functional groups can be expressed as

$$
\begin{gathered}
\mathrm{AH}_{Z_{a}-(n-1)}^{(n-1)-} \Leftrightarrow \mathrm{AH}_{Z_{a}-n}^{n-}+\mathrm{H}^{+}, K_{a, n}, n=1,2, \ldots, Z_{a}, \\
\mathrm{BH}_{Z_{b}-(n-1)}^{\left[Z_{\left.Z^{-}-(n-1)\right]+}\right.} \Leftrightarrow \mathrm{BH}_{Z_{b}-n}^{\left(Z_{b}-n\right)+}+\mathrm{H}^{+}, K_{b, n}, n=1,2, \ldots, Z_{b},
\end{gathered}
$$

where $Z_{a}$ is the number of dissociable protons of an acidic group $\mathrm{AH}_{Z_{a}}$, and $Z_{b}$ is the number of absorbable protons of a basic group B, $K_{a, n}, n=1,2, \ldots, Z_{a}$, and $K_{b, n}, n=1$, $2, \ldots, Z_{b}$, are the corresponding equilibrium constants. Denote $x_{k}, k=1,2, \ldots, Z_{a}$, and $y_{k}, k=1,2, \ldots, Z_{b}$, as the concentrations of $\mathrm{AH}_{Z_{a}-1}^{-}, \ldots, \mathrm{AH}^{Z_{a}^{-}}$and $\mathrm{BH}_{Z_{b}-1}^{\left(Z_{b}^{-1}\right)+}, \ldots$, $\mathrm{B}$ respectively. We have

$$
K_{v, 1}=\frac{u_{1} C_{\mathrm{H}^{+}}}{\left(N_{v}^{i=1}-\sum_{m=1}^{Z_{v}} u_{m}\right)}, \quad \begin{gathered}
v=\mathrm{a} \text { and } u=x, \text { or } \\
v=\mathrm{b} \text { and } u=y,
\end{gathered}
$$

where $C_{\mathrm{H}^{+}}$is the concentration of $\mathrm{H}^{+}$which is calculated by

$$
C_{\mathrm{H}^{+}}=C_{\mathrm{H}^{+}}^{0} \exp (-\psi)
$$


$C_{\mathrm{H}^{+}}^{0}$ is the bulk concentration of $\mathrm{H}^{+}$, and

$$
u_{n}=\frac{u_{n-1} K_{v, n}}{C_{\mathrm{H}^{+}}}, n=2,3, \ldots, Z_{v}, \quad \begin{aligned}
& v=a \text { and } u=x, \text { or } \\
& v=b \text { and } u=y .
\end{aligned}
$$

Here, $N_{v}^{i=1}, v=a, b$, are the concentrations of acidic and basic functional groups in the membrane phase, respectively. These expressions yield

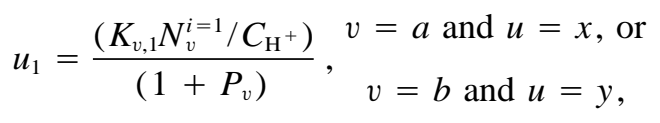

where

$$
P_{v}=\sum_{m=1}^{Z_{v}}\left[\prod_{j=1}^{m} \frac{K_{v, j}}{C_{\mathrm{H}^{+}}}\right], v=a, b
$$

Equations [4] and [5a] lead to

$$
\begin{aligned}
& u_{n}=\frac{N_{v}^{i=1}}{1+P_{v}} \prod_{j=1}^{n} \frac{K_{v, j}}{C_{\mathrm{H}^{+}}}, \\
& n=2,3, \ldots Z_{v}, \quad v=a \text { and } u=x \text {, or } \\
& \quad v=b \text { and } u=y .
\end{aligned}
$$

The concentrations of negative fixed charges, $N_{-}^{i=1}$, and positive fixed charges, $N_{+}^{i=1}$, are, respectively,

$$
N_{-}^{i=1}=\sum_{n=1}^{Z_{a}} n x_{n}=\frac{N_{a}^{i=1} Q_{a}}{1+P_{a}}
$$

and

$$
\begin{aligned}
N_{+}^{i=1} & =Z_{b}\left(N_{b}^{i=1}-\sum_{n=1}^{Z_{b}} y_{n}\right)+\sum_{n=1}^{Z_{b}-1}\left[\left(Z_{b}-n\right) y_{n}\right] \\
& =Z_{b} N_{b}^{i=1}-\sum_{n=1}^{Z_{b}}\left(n y_{n}\right) \\
& =N_{b}^{i=1}\left(Z_{b}-\frac{Q_{b}}{1+P_{b}}\right)
\end{aligned}
$$

where

$$
Q_{v}=\sum_{n=1}^{z_{v}}\left[n \prod_{j=1}^{n} \frac{K_{v, j}}{C_{\mathrm{H}^{+}}}\right], v=a, b
$$

Electrostatic Interaction Force

The electrical interaction force between particle and surface $F_{\mathrm{R}}$ is calculated by

$$
\begin{gathered}
\frac{F_{\mathrm{R}}}{b n_{b}^{0} k_{\mathrm{B}} T}=\frac{1}{b}\left(e^{b \psi}-1\right)+\frac{1-\eta}{a}\left(e^{-a \psi}-1\right) \\
+\frac{\eta}{c}\left(e^{-c \psi}-1\right)-\left[\frac{(a+b)+(c-a) \eta}{2}\right]\left(\frac{d \psi}{d X}\right)^{2} \\
+i \int_{\psi}^{0} N d \psi, i=0,1
\end{gathered}
$$

The numerical procedure for the evaluation of $F_{\mathrm{R}}$ is summarized in the Appendix.

\section{DISCUSSION}

The general expression, [9], is an extension of the classic result for the case of two identical rigid surfaces (10). The last term on the right-hand side of this expression is due to the existence of a membrane phase. For rigid surfaces, it vanishes $(i=0)$. Furthermore, for two identical rigid surfaces, $(d \psi / d X)=0$ at the midplane between two surfaces, and $\psi=\psi_{\mathrm{m}}$ at this plane. In this case, [9] reduces to

$$
\begin{aligned}
\frac{F_{\mathrm{R}}}{b n_{b}^{0} k_{\mathrm{B}} T}=\frac{1}{b}\left(e^{b \psi_{\mathrm{m}}}-1\right)+\frac{1-\eta}{a}( & \left.e^{-a \psi_{\mathrm{m}}}-1\right) \\
& +\frac{\eta}{\mathrm{c}}\left(e^{-c \psi_{\mathrm{m}}}-1\right) .
\end{aligned}
$$

This is consistent with the result of Kuo and Hsu (11). For an $a: b$ electrolyte solution, the repulsion force can be obtained by substituting $\eta=0$ into [10]. Moreover, if the electrolyte is symmetric, $a=b=z$, [10] becomes

$$
\frac{F_{\mathrm{R}}}{z n^{0} k_{\mathrm{B}} T}=2\left[\cosh \left(\frac{z e \psi_{\mathrm{m}}}{k_{\mathrm{B}} T}\right)-1\right] \text {, }
$$

where $n^{0}$ is the number concentration of ionic species in the bulk liquid phase. This is consistent with the classic result (10).

In a study of the electrostatic interaction between two amphoteric surfaces, Chan and co-workers $(4,5)$ considered the following mechanism for proton transfer:

$$
\begin{aligned}
\mathrm{AH}^{2+} & =\mathrm{AH}+\mathrm{H}^{+} \\
\mathrm{AH} & =\mathrm{A}^{-}+\mathrm{H}^{+} .
\end{aligned}
$$

This is an example of [2a]. The following compounds all contain multiproton functional groups (12): $\mathrm{H}_{3} \mathrm{AsO}_{4}\left(K_{a, 1}\right.$ $\left.=6.0 \times 10^{-3}, K_{a, 2}=1.05 \times 10^{-7}, K_{a, 3}=3.0 \times 10^{-12}\right)$, 


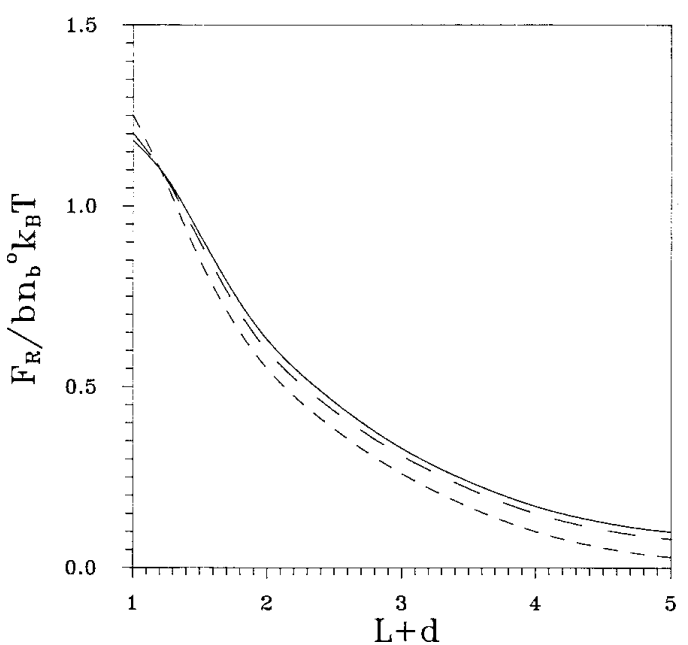

FIG. 2. Variation in the electrostatic interaction force between particle and surface as a function of separation distance at various $\eta$ for the case pH 7 and $d=1$. - , $\eta=0 ;---, \eta=0.05$; ---, $\eta=0.1$. Parameters: $\varepsilon_{\mathrm{r}}=78, T=298.15 \mathrm{~K}, \psi_{0}=-1$, ionic strength $=10^{-3} M, N_{a}^{i=1}=N_{b}^{i=1}$ $=10^{-3} M, Z_{a}=Z_{b}=3, \mathrm{p} K_{a, n}=(2+n), \mathrm{p} K_{b, n}=(4+n), n=1,2,3$, $a=b=1$, and $c=2$.

$\mathrm{H}_{3} \mathrm{PO}_{4}\left(K_{a, 1}=7.11 \times 10^{-3}, K_{a, 2}=6.34 \times 10^{-8}, K_{a, 3}=\right.$ $\left.4.2 \times 10^{-13}\right), \mathrm{HOOC}(\mathrm{OH}) \mathrm{C}\left(\mathrm{CH}_{2} \mathrm{COOH}\right)_{2}\left(K_{a, 1}=7.45 \times\right.$ $\left.10^{-4}, K_{a, 2}=1.73 \times 10^{-5}, K_{a, 3}=4.02 \times 10^{-7}\right), \mathrm{H}_{4} \mathrm{Y}\left(K_{a, 1}\right.$ $=1.0 \times 10^{-2}, K_{a, 2}=2.1 \times 10^{-3}, K_{a, 3}=6.9 \times 10^{-7}, K_{a, 4}$ $\left.=5.5 \times 10^{-11}\right)$, and $\mathrm{NH}_{2} \mathrm{C}_{2} \mathrm{H}_{4} \mathrm{NH}_{2}\left(K_{1}=8.5 \times 10^{-5}, K_{2}\right.$ $\left.=7.1 \times 10^{-8}\right)$. The first two compounds are capable of forming a coordinate bond with membrane materials, and the rest capable of undergoing a dehydration reaction with membrane materials. The dissociation of the functional groups of the membrane thus obtained can be described by [2a] and [2b].

Figure 2 shows the simulated variation in the electrostatic interaction force between particle and surface as a function of separation distance at various $\eta$. Note that the particle carries a net negative fixed charges. Figure 2 suggests that for a fixed $\eta$, the repulsion force decreases with $(L+d)$, as expected. For medium to large $(L+d)$, the repulsion force decreases with $\eta$. Since $a=b=1$, and $c=2$, this indicates that the presence of multivalent cations in the liquid phase has the effect of reducing the repulsion force. However, for small values of $(L+d)$, the reverse is true. In other words, if $(L+d)$ is greater than a critical value, the presence of multivalent cations in the liquid phase has the effect of reducing the repulsion force between particle and surface. On the other hand, if $(L+d)$ is smaller than this critical value, the presence of multivalent cations will increase the repulsion force between particle and surface. Similar result was obtained for the case of two rigid surfaces, one of which bears ionizable functional groups (4). The behavior for small $(L+d)$ was interpreted as the requirement for continuous reequilibration of the functional groups on the surface of particle and the screening out of those overloaded multivalent cations in the interacting region between the particle and the surface, as the separation distance decreases.

The variation in the electrostatic interaction force between particle and surface as a function of separation distance at various $\mathrm{pH}$ is illustrated in Fig. 3. For a fixed separation distance between particle and surface, the repulsion force is found to increase with $\mathrm{pH}$. This is because the smaller the $\mathrm{pH}$, the less the degree of dissociation of the acidic groups in the membrane phase, and the easier for $\mathrm{H}^{+}$to bind to the basic groups. As a result, the amount of negative fixed charges in the membrane phase decreases, and the repulsion force decreases.

Figure 4 simulates the variation in the electrostatic interaction force between particle and surface as a function of separation distance at constant total number of functional groups for various $d$. As can be seen from this figure, the repulsion force decreases with $d$. This is because at a constant total number of functional groups the thinner the membrane, the more concentrated the fixed charges, and the greater the electrical interaction. It is interesting to note that if the dimensionless thickness of membrane is smaller than a critical value $d_{\mathrm{c}}$, the distribution of repulsion force as a function of separation distance has a maximum. This is mainly due to the regulation behavior of the membrane. If $d$ is greater than $d_{\mathrm{c}}$, the interaction force decreases monotonically with the separation distance.

\section{APPE NDIX}

The numerical procedure for the estimation of the electrostatic interaction force between particle and surface is summarized below.

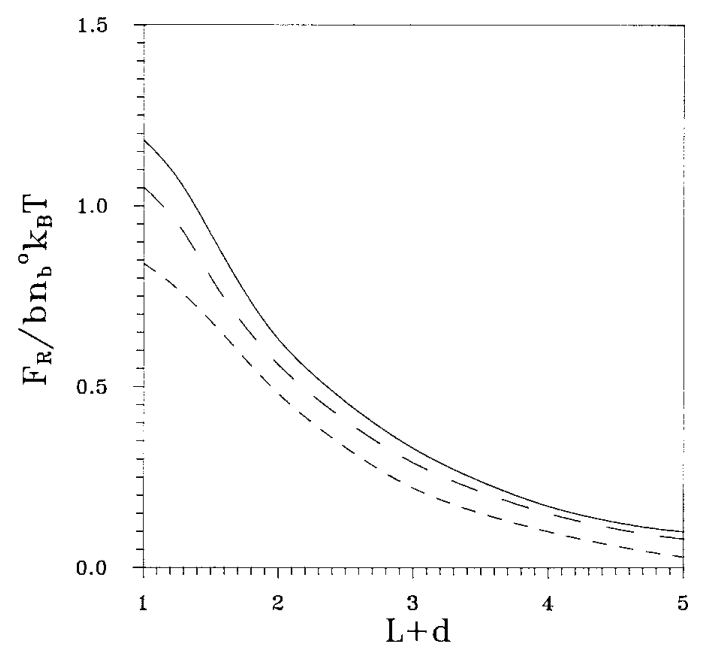

FIG. 3. Variation in the electrostatic interaction force between particle and surface as a function of separation distance at various $\mathrm{pH}$ for the case $\eta=0$ and $d=1$. - , $\mathrm{pH} \mathrm{7;---,} \mathrm{pH} 6.5 ;---, \mathrm{pH}$ 6. Parameters are as described in the legend to Fig. 2. 


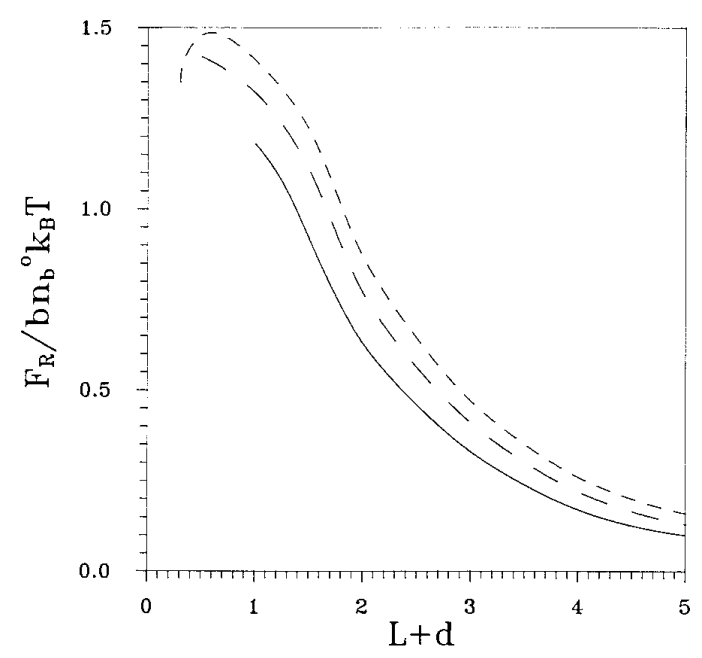

FIG . 4. Variation in the electrostatic interaction force between particle and surface as a function of separation distance at various $d$ for the case $\eta$ $=0$ and $\mathrm{pH} \mathrm{7.}-, d=1 ;---, d=0.5 ;---, d=0.3$. Parameters are as described in the legend to Fig. 2.

Step 1. For a given $L$, [1a] with $i=0$ is solved subject to [1e] and an estimated dimensionless differential potential at the rigid surface, $\psi_{\mathrm{s}, \mathrm{g}}^{\prime}$. This yields the potential distribution in the double layer.

Step 2. Solving [1a] with $i=1$ subject to [1c] and [1d], the potential distribution in the membrane phase is obtained.
We check whether [1b] is satisfied. If it is not satisfied, return to step 1 with a newly estimated $\psi_{\mathrm{s}, \mathrm{g}}^{\prime}$.

Step 3. $F_{\mathrm{R}}$ is estimated through [9].

\section{ACKNOWLEDGMENT}

This work is supported by the National Science Council of the Republic of China under Grant NSC84-2214-E002-030.

\section{REFERENCES}

1. Weiss, L., and Harlos, J. P., J. Theor. Biol. 37, 1169 (1972).

2. Ninham, B. W., and Parsegian, V. A., J. Theor. Biol. 31, 405 (1971).

3. Healy, T. W., Chan, D., and White, L. R., Pure Appl. Chem. 52, 1207 (1980).

4. Chan, D., Perram, J. W., White, L. R., and Healy, T. W., J. Chem. Soc. Faraday Trans. I 71, 1046 (1975).

5. Chan, D., Healy, T. W., and White, L. R., J. Chem. Soc. Faraday Trans. I 72, 2844 (1976).

6. Prieve, D. C., and Ruckenstein, E., J. Theor. Biol. 56, 205 (1976).

7. Ohshima, H., and Ohki, S., Biophys. J. 47, 673 (1985).

8. Seaman, G. V., in "The Red Blood Cells"' (D. M. Sergenor, Ed.), Vol. 2, p. 1136. Academic Press, New York, 1975.

9. Kawahata, S., Ohshima, H., Muramatsu, N., and Kondo, T., J. Colloid Interface Sci. 138, 182 (1990).

10. Hiemenz, P. C., "Principles of Colloid and Surface Chemistry,"' 2nd ed. Dekker, New York, 1986.

11. Kuo, Y. C., and Hsu, J. P., J. Colloid Interface Sci. 156, 250 (1993).

12. Meites, L., "Handbook of Analytical Chemistry,"' p. 1. McGraw-Hill, New York, 1963. 